\title{
Attentional, visual, and emotional mechanisms of face processing proficiency in Williams syndrome
}

\author{
Rashmi Gupta* \\ School of Psychology, Bangor University, Bangor, UK \\ *Correspondence: rash_cogsci@yahoo.com
}

\section{A commentary on}

The fusiform face area is enlarged in Williams syndrome

by Golarai, G., Hong, S., Haas, B. W., Galaburda, A. M., Mills, D. L., Bellugi, U., Grill-Spector, K., and Reiss, A. L. (2010). J. Neurosci. 30, 6700-6712.

Williams syndrome (WS) is a disorder of neural development caused by a micro deletion of about 26 genes from the long arm of chromosome 7q11.23 (Golarai et al., 2010). Individuals with WS were found to be are as good as typically developed (TD) healthy participants on Benton Recognition test, which is used to measure face recognition proficiency (Golarai et al., 2010). Golarai et al. (2010) used functional magnetic resonance imaging to investigate neural substrates underlying face-identity recognition in WS. They compared group differences in absolute fusiform area (FFA) size and also FFA size relative to the anatomical size of the fusiform gyrus, response amplitudes to faces and objects between WS and TD adults.

Volumes of the FFA in WS and TD participants were found to be larger in absolute terms in WS than TD participants in both hemispheres. Golarai et al. (2010) raised the possibility to explain face recognition proficiency in WS in terms of larger FFA volume, a developmental perspective of FFA volume, and genetic factors underlying FFA's larger volume in WS. Golarai et al. (2010) and many other studies focus on the FFA but that its functional role in face recognition is far from clear. The role of FFA in face-identity (and emotion) recognition must be viewed against the background of its connections to a number of other areas involved in attention and emotion processing.

Golarai et al. (2010) did not discuss a possible role of attention or attentional networks in processing of faces in WS individuals. It has been suggested that face processing/emotional processing and attentional processing activates similar brain regions (Pessoa, 2009). Pessoa (2009) suggested that the attentional network involves fronto-parietal regions, including the middle frontal gyrus, anterior cingulate cortex (ACC), inferior frontal gyrus, and anterior insula, which also involved in the processing of faces and emotional information. Overlapping brain networks of face and attentional processing suggested the role of attention in face processing. This explanation is fits with electrophysiological reports of atypical larger N200 were observed in WS, which was found to be correlated with performance on the Benton test (Mills et al., 2000). Larger N200 reflect increased attention to faces. In addition, the WS group showed disproportionate increases in volume and gray matter density in some brain regions of attention (ACC, anterior insula) as well as other regions including amygdala, orbital prefrontal cortices, and superior temporal gyrus, which are known to participate in emotion and face processing. The larger volume and gray matter density of the attentional networks may help WS individuals to attend faces and may underlie the islands of preserved skills of face processing in WS. This explanation fits with findings of Golarai et al. (2010) who showed that WS individuals are efficient in face recognition. This neuroanatomical link between attention and face processing was not made explicit by Golarai et al. (2010), which has important implications for the role of attentional mechanisms serving face processing in WS individuals.

Alternatively, enhancement of face processing in WS might be aided by visual input received by primary visual cortex. For example, Galaburda and Bellugi (2000) suggested that the average size of cortical neurons was found to be greater in primary visual cortex in WS individuals than in control brains, coupled with normal cell packing density, which could result in enhanced visual input of face processing in WS brains.
This enhancement in face processing with bigger FFA may results in better processing of faces but not for objects.

Increased activation in amygdala was observed for processing of happy faces compared to fearful faces in WS individuals (Haas et al., 2010). Higher activation for happy faces and less activation for fearful faces in WS individuals indicate a possibility that WS individuals may see the fearful face as happy/positive face or approachable face as they can not inhibit an approach response to fearful/negative faces. This explanation is consistent with the finding that WS individuals show less activation in the amygdala during the processing of fearful/threatening face as compared to fearful/threatening scenes (Meyer-Lindenberg et al., 2005). Medial-prefrontal cortex (MPFC) and orbito-frontal cortex (OFC) are densely interconnected with amygdala and dorsolateral-prefrontal cortex (DLPFC) and have been implicated in the regulation of amygdala function, social cognition, and representation of social knowledge. Meyer-Lindenberg et al. (2005) found that WS individuals showed no activation in OFC during fearful/threatening face processing. In addition, no functional connection was observed of OFC with amygdala or DLPFC with in WS, which provide evidence of social disinhibition and impairments in adjusting behavior according to social clues in WS individuals. MPFC has been associated with empathy, representation of social knowledge, and integration of emotional information about others and self. MPFC region was found to be persistently activated in WS individuals during fearful/ threatening face processing, which maps well on phenotypic characteristics of relative social strengths with WS, such as increased empathy.

To understand the complete picture of face processing proficiency in WS, it is also important to consider the above mentioned 
attentional, visual, and emotional mechanisms of face processing in WS. Future work directed toward investigating the functional consequences of FFA size in face processing with longitudinal studies of young children with WS that will be of great important in understanding the mechanisms of cortical specialization during normal and atypical development.

\section{REFERENCES}

Galaburda, A. M., and Bellugi, U. (2000). Multi-level analysis of cortical neuroanatomy in Williams syndrome. J. Cogn. Neurosci. 12, 74-88.
Golarai, G., Hong, S., Haas, B. W., Galaburda, A. M., Mills, D. L., Bellugi, U., Grill-Spector, K., and Reiss, A. L. (2010). The fusiform face area is enlarged in Williams syndrome. J. Neurosci. 30, 6700-6712.

Haas, B. W., Hoeft, F., Searcy, Y. M., Mills, D., Bellugi, U., and Reiss, A. (2010). Individual differences in social behavior predict amygdala response to fearful facial expressions in Williams syndrome. Neuropsychologia $48,1283-1288$.

Meyer-Lindenberg, A., Hariri, A. R., Munoz, K. E., Mervis, C. B., Mattay, V. S., Morris, C. A., and Berman, K. F. (2005). Neural correlates of genetically abnormal social cognition in Williams syndrome. Nat. Neurosci. 8, 991-993.

Mills, D. L., Alvarez, T. D., St George, M., Appelbaum, L. G. Bellugi, U., and Naville, H. (2000).III.Electrophysiological studies of face processing in Williams syndrome. J. Cogn. Neurosci. 22, 197-207.

Pessoa, L. (2009). How do emotion and motivation direct executive control? Trends Cogn. Sci. 13, 160-166.

Received: 18 January 2011; accepted: 26 March 2011; published online: 05 April 2011.

Citation: Gupta R (2011) Attentional, visual, and emotional mechanisms of face processing proficiency in Williams syndrome. Front. Behav. Neurosci. 5:18. doi: 10.3389/ fnbeh.2011.00018

Copyright $\odot 2011$ Gupta. This is an open-access article subject to a non-exclusive license between the authors and Frontiers Media SA, which permits use, distribution and reproduction in other forums, provided the original authors and source are credited and other Frontiers conditions are complied with. 